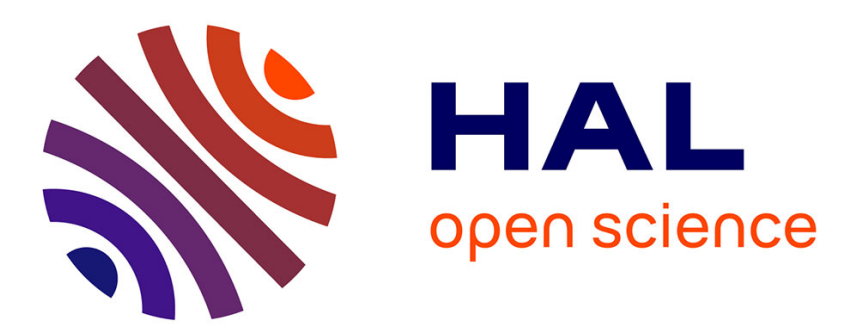

\title{
Vision-Based Altitude, Position and Speed Regulation of a Quadrotor Rotorcraft
}

Eduardo Rondon, Luis-Rodolfo Garcia Carrillo, Isabelle Fantoni

\section{To cite this version:}

Eduardo Rondon, Luis-Rodolfo Garcia Carrillo, Isabelle Fantoni. Vision-Based Altitude, Position and Speed Regulation of a Quadrotor Rotorcraft. IEEE/RSJ International Conference on Intelligent Robots and Systems (IROS 2010), Oct 2010, Taipei, Taiwan. pp.628-633. hal-00522137

\section{HAL Id: hal-00522137 \\ https://hal.science/hal-00522137}

Submitted on 29 Sep 2010

HAL is a multi-disciplinary open access archive for the deposit and dissemination of scientific research documents, whether they are published or not. The documents may come from teaching and research institutions in France or abroad, or from public or private research centers.
L'archive ouverte pluridisciplinaire HAL, est destinée au dépôt et à la diffusion de documents scientifiques de niveau recherche, publiés ou non, émanant des établissements d'enseignement et de recherche français ou étrangers, des laboratoires publics ou privés. 


\title{
Vision-Based Altitude, Position and Speed Regulation of a Quadrotor Rotorcraft
}

\author{
Eduardo Rondon, Luis-Rodolfo Garcia-Carrillo, Isabelle Fantoni
}

\begin{abstract}
In this paper, we address the hover flight and speed regulation of a quadrotor rotorcraft to perform autonomous navigation. For this purpose, we have developed a vision system which estimates the altitude, the lateral position and the forward speed of the engine during flights. We show that the visual information allows the construction of control strategies for different kinds of flying modes: hover flight, forward flight at constant speed. A hierarchical control strategy is developed and implemented. The local stabilization of the vehicle is proven. Experimental autonomous flight was successfully achieved which validates the visual algorithm and the control law.
\end{abstract}

\section{INTRODUCTION}

The development of mini UAVs and micro UAVs has increased during last years. The capability to fly indoor as well as outdoor makes this kind of autonomous systems very suitable for a wide range of applications either military or civilian. In the military field, we can mention applications such as delivering survival kits, communication link, risk evaluation of sensible areas. In the civilian field, we can find delicate applications such weather monitoring, cartography, traffic monitoring, search and rescue missions, surveillance operations and remote sensing. Accurate navigation and efficient control of the vehicle are essential to accomplish successfully such missions. Many efforts have been done in the development of these two domains, and at present, real-time embedded stabilization has been already proved (for example in [1], [2]). In the other hand, autonomous navigation keeps motivating new research areas. Moreover, for a complete implementation of fully autonomous UAVs, the vehicle must be capable of navigating through unknown environments.

Image-based navigation arises as an attractive solution, small low cost cameras attached to the vehicle provide detailed information about the UAV motion and the environment structure. Due to this interaction between the sensor with its surroundings, new techniques can be developed permitting the vehicle to navigate within clustered environments. In recent years, vision has become an "ideal ally" to UAVs. Nowadays, vision algorithms lead to interesting and promising results. Algorithms based in monocular vision and others based in stereo vision, are proposed as solutions to different kinds of missions or environments. Johnson et al. [3] and Yu et al. [4] used stereo vision to control the

This work was supported by the French Research Foundation for Aeronautics and Space

E. Rondon, L.R. Garcia and I.Fantoni are with the Universite de Technologie de Compiègne - CNRS UMR 6599 Heudiasyc, BP 20529, 60205 Compiègne Cedex, France Eduardo.Rondonehds.utc. fr height of an helicopter, and to developed an autonomous landing strategy. In [5], a stereo system is combined with a monocular camera in order to navigate and avoid obstacles. A system composed of two cameras is presented in [6] to stabilize the position of a convertible mini VTOL, each camera is used to control different dynamics of the aircraft. Monocular systems have also been implemented, Guenard et al. [7] used visual-servo control techniques in order to stabilize the position of a quadrotor.

New control strategies inspired on animals and insects behavior, have reached the domain of UAVs research. Chahl et al. [8] proposed a landing strategy where the honeybee uses optical flow for landing. In [9], an optical flow-based controller was implemented to land a quadrotor. Terrain following missions using optical flow have also been implemented in [10], [11] and [12]. Serres et al. [13] developed an optical flow-based autopilot to regulate speed and avoid lateral obstacles. Zufferey et al. [14] built a $30 \mathrm{~g}$ microflyer which avoids obstacles using optical flow.

In this paper, we propose a monocular vision system permitting the control of the altitude with the objective of stabilizing the position and regulating the speed of a quadrotor rotorcraft using optical flow. The purpose of our approach is to eliminate the position drift when hovering, and to ensure accurate translation. These two properties are necessary to build an autonomous navigation system. If the position drift is compensated, the hover flight could be used by the system as an intermediary between different flying modes which are adapted to different conditions of the environment. Moreover, speed regulation is used to create the different flying modes, for instance, only lateral displacement and only forward displacement. In order to correctly use the optical flow, we also developed a vision-based altitude controller. The combination of these three vision-based controls allows the vehicle to navigate in a realistic application. We illustrate this by testing our system in two different kinds of missions: position holding on a road segment and road following.

The body of this paper is organized as follows. In section II, we describe the implemented visual system. Section III specifies the dynamical model of the helicopter. In section IV, the proposed visual-based control law is discussed. Section V describes the experimental setup and shows the results obtained during autonomous flight. We conclude our work in section VI.

\section{VISUAL SySTEM}

The overall system consists of the rotorcraft, the downward looking camera attached to the vehicle, and the road 
model (see Figure 1). The system is defined as follows:

- The rotorcraft

Evolving in space, with 6DOF, it is related to it's own body coordinate system $\left(X_{B}, Y_{B}, Z_{B}\right)$ and to the fixed inertial frame $\left(X_{I}, Y_{I}, Z_{I}\right)$.

- The downward camera

Attached to the rotorcraft, the camera undergoes the same movement. Any motion sensed, represents a displacement of the helicopter.

- The road model represented in Figure 1 and Figure 2, is a dual carriageway road, with a crash barrier separating both carriageway.

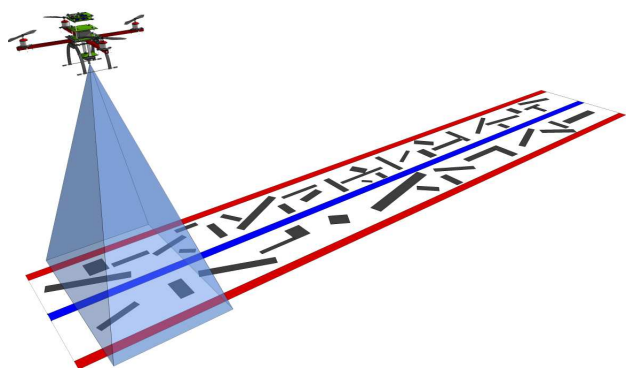

Fig. 1. System configuration

The visual algorithm computes an estimate of altitude, the position and the pseudo-velocities. The altitude and some position information of the engine are deduced by extracting the road zone from the image, the pseudo speed is estimated by means of optical flow computation. All information sensed by the camera is related to the image plane, we show that this information is rich enough to control the vehicle.

\section{A. Extracting Road Zone}

The road zone is computed by a combination of line detection and color segmentation processes. The first step is to recognize the color zones, this is achieved by a classical color segmentation algorithm. The segmentation is done in the $R G B$ encoding format. A threshold level $\tau_{r}$ between the three colors defines the red color, and a threshold $\tau_{b}$ defines the blue color. Once the segmentation task is done, the image is passed through a series of gaussian filters, and then the algorithm continues with a line detection process based on a Hough's Transform. In the final stage of this algorithm, both results are combined to estimate the points $x_{l_{1}}^{i}, x_{l_{2}}^{i}, x_{\text {min }}^{i}, x_{\text {max }}^{i}$, $x_{r_{1}}^{i}$ and $x_{r_{2}}^{i}$. These points are image projections of the world coordinates $x_{l_{1}}, x_{l_{2}}, x_{\min }, x_{\max }, x_{r_{1}}$ and $x_{r_{2}}$ representing the left border, central separation and right border of the road (see Figure 2). With all extracted image points, the road zone is totally defined and the altitude and the position can be estimated.

\section{B. Altitude and Position}

Once the road information has been extracted, position and altitude estimates can be built. The altitude estimation is done using both lines passing through the points $x_{l_{1}}^{i}, x_{l_{2}}^{i}$,

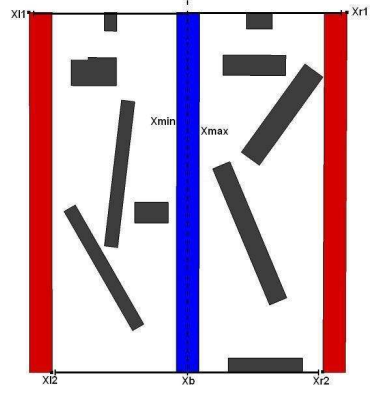

Fig. 2. Road Model

$x_{r_{1}}^{i}, x_{r_{2}}^{i}$. The importance of using these points is to have an estimate independent of the yaw angle of the vehicle with respect to the inertial frame (road coordinate system). We then define the following variables as

$$
\begin{aligned}
x_{l_{m}}^{i} & =\frac{x_{l_{2}}^{i}+x_{l_{1}}^{i}}{2} \\
x_{r_{m}}^{i} & =\frac{x_{r_{2}}^{i}+x_{r_{1}}^{i}}{2} \\
\omega & =x_{r_{m}}^{i}-x_{l_{m}}^{i}
\end{aligned}
$$

where $x_{l_{m}}^{i}, x_{r_{m}}^{i}$ represent the border's centroids of the left and right carriageway of the road, respectively. Those points are in the image plane, and are related to the world points by the projective camera relations. If we consider a weak projection (all points having the same depth $z$ ), then we can write

$$
\begin{aligned}
x_{l_{m}}^{i} & =\frac{f x_{l}}{z} \\
x_{r_{m}}^{i} & =\frac{f x_{r}}{z}
\end{aligned}
$$

Replacing equations (4) and (5) in equation (3), we have

$$
\omega=\frac{f\left(x_{r}-x_{l}\right)}{z}=\frac{f W}{z}
$$

where $W$ is the distance between the left and right boarders, i.e. the width of the road. Then,

$$
\frac{1}{\omega}=K_{\text {im }} z
$$

where $K_{i m}$ is a constant depending only on the focal length of the camera and the width of the road, and $z$ is the altitude of the engine. We can note from equation (7) that the control of the inverse of the image-based variable $\omega$ is equivalent to control the altitude $z$ of the rotorcraft. The lateral position of the helicopter can also be deduced from the road extraction points. The lines passing through the points $x_{\text {min }}^{i}, x_{\text {max }}^{i}$ define the new centroid $x^{i}$ (which is the projection of the point $x_{b}$, see figure 2). The objective is to bring the image point $x^{i}$ to the center of the image, which is close enough to the principal point of the image. This point can be considered as the projection of the center of gravity of the vehicle, because the center of the camera and the center of the rotorcraft coordinate system are aligned. Therefore, we can construct the new image-based variable as follows:

$$
\varsigma=\frac{f\left(x-x_{b}\right)}{z}
$$


Here $x$ is the position of the center of gravity of the rotorcraft in the inertial frame, $x_{b}$ is central line centroid, and $\varsigma$ is the image-based control variable. A study of the time derivative is needed in order to measure the impact of an altitude variation on this variable.

$$
\begin{aligned}
& \dot{\boldsymbol{\zeta}}=\frac{f}{z} \dot{x}-\frac{f\left(x-x_{b}\right)}{z^{2}} \dot{z} \\
& \dot{\boldsymbol{\zeta}}=\frac{f}{z} \dot{x}+\frac{\left(x-x_{b}\right)}{z W} \dot{\omega}
\end{aligned}
$$

where $\dot{x}$ is the lateral speed of the engine, $\dot{\zeta}$ is the time derivative of the image-based lateral position variable, and $\dot{\omega}$ is the time derivative of the image-based altitude variable. From equation (10) we can see that a prior stabilization of $\frac{1}{\omega}$ and $\dot{\omega}$ is a necessary requirement to fulfill a good lateral position stabilization. Once the altitude is maintained constant, the time derivative $\dot{\zeta}$ will only depend of the lateral speed of the engine, any other contribution will be considered as noise. Finally, when the altitude is stabilized $(\dot{\omega}=0)$, the time derivatives of the image-based variables become $\dot{\boldsymbol{\zeta}}=\frac{f}{z} \dot{x}$.

\section{Pseudo-Speeds}

We consider the camera moving with respect to a rigid scene. The velocities and rotation rates of the camera in the inertial frame are $\left(V_{x}, V_{y}, V_{z}\right)$ and $\left(\omega_{x}, \omega_{y}, \omega_{z}\right)$. To accurately estimate the pseudo-speeds of the engine we define a tracking zone inside the road model. The tracking zone is defined in a way that the point $x^{i}=\frac{f x_{b}}{z}$ is the centroid of the zone. The optical flow computed at point $\left(x_{k}^{i}, y_{k}^{i}\right)$ is composed of a translational and a rotational part as

$$
\left[\begin{array}{c}
O F_{x_{k}^{i}} \\
O F_{y_{k}^{i}}
\end{array}\right]=T_{O F_{k}}+R_{O F_{k}}
$$

with the translational part

$$
T_{O F_{k}}=\frac{1}{z}\left[\begin{array}{ccc}
-f & 0 & x_{k}^{i} \\
0 & -f & y_{k}^{i}
\end{array}\right]\left[\begin{array}{c}
V_{x} \\
V_{y} \\
V_{z}
\end{array}\right]
$$

and the rotational part

$$
R_{O F_{k}}=\left[\begin{array}{ccc}
\frac{x_{k}^{i} y_{k}^{i}}{f} & -\left(f+\frac{\left(x_{k}^{i}\right)^{2}}{f}\right) & y_{k}^{i} \\
\left(f+\frac{\left(y_{k}^{i}\right)^{2}}{f}\right) & -\frac{x_{k}^{i} y_{k}^{i}}{f} & -x_{k}^{i}
\end{array}\right]\left[\begin{array}{l}
\omega_{x} \\
\omega_{y} \\
\omega_{z}
\end{array}\right]
$$

The spatial mean of the optical flow (mean of the optical flow on all tracked points) is then (see [15])

$$
\begin{gathered}
\overline{O F}_{x}=\bar{V}_{O F_{x}}+K_{x}^{x} \bar{V}_{O F_{z}}+\bar{R}_{O F_{x}} \\
\overline{O F}_{y}=\bar{V}_{O F_{y}}+K_{y}^{y} \bar{V}_{O F_{z}}+\bar{R}_{O F_{y}}
\end{gathered}
$$

Using the results from [15], the rotational optical flow is compensated and the pseudo-speeds $\bar{V}_{O F_{x}}, \bar{V}_{O F_{y}}$ and $\bar{V}_{O F_{z}}$ are deduced. Since the camera system and the helicopter share the same movements, we can write

$$
\begin{array}{r}
\bar{V}_{O F_{x}}=-\frac{f \dot{x}}{z}=-\dot{\zeta} \\
\bar{V}_{O F_{y}}=-\frac{f \dot{y}}{z}=-\dot{y}^{i} \\
\bar{V}_{O F_{z}}=\frac{\dot{z}}{z}
\end{array}
$$

where $(\dot{x}, \dot{y}, \dot{z})$ is the speed vector of the rotorcraft center of gravity. From equations (7), (15) and (16) we note that the visual system allows altitude, position and speed control of the rotorcraft using only the image-based variables $(\zeta, \dot{\zeta})$, $\left(\frac{1}{\omega},\left(\frac{\dot{1}}{\omega}\right)\right)$ and $\dot{y}^{i}=-\bar{V}_{O F_{y}}$.

\section{ROTORCRAFT DYNAMICS}

The rotorcraft with an embedded camera, that we use

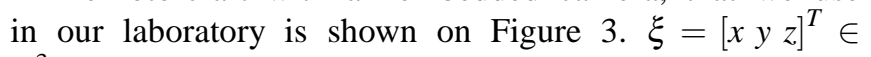
$\mathbb{R}^{3}$ denotes the position of the vehicle's center of gravity, relative to the inertial frame, and $\eta=\left[\begin{array}{lll}\phi & \theta & \psi\end{array}\right]^{T} \in \mathbb{R}^{3}$ are the three Euler angles (roll, pitch and yaw), which represent the orientation of the vehicle. The model of the rotorcraft dynamics is obtained from Euler-Lagrange equations (see [1])

$$
\begin{array}{r}
m \ddot{x}=-u \sin \phi \\
m \ddot{y}=u \cos \phi \sin \theta \\
m \ddot{z}=u \cos \theta \cos \phi-m g \\
\ddot{\theta}=\tilde{\tau}_{\theta} \\
\ddot{\phi}=\tilde{\tau}_{\phi} \\
\ddot{\psi}=\tilde{\tau}_{\psi}
\end{array}
$$

where $u, \tilde{\tau}_{\theta}, \tilde{\tau}_{\phi}, \tilde{\tau}_{\psi}$ are the force and torque inputs.

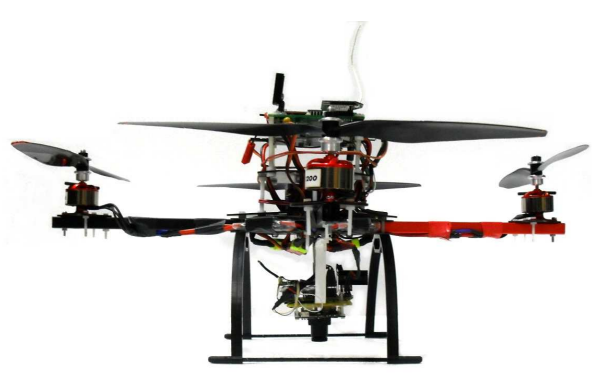

Fig. 3. Four rotor helicopter with a camera

\section{Control Strategy}

In this study, a hierarchical control strategy was implemented. Considering the time scale separation between the navigation control and the low level autopilot, the dynamical model can be seen as a series of nested subsystems. The first two subsystems to be stabilized are the altitude and the yaw dynamics.

\section{A. Altitude subsystem}

Considering equation (20), the altitude dynamics can be represented as follows

$$
\ddot{z}=\cos \theta \cos \phi\left(\frac{u}{m}-g\right)
$$

Then, the altitude can be stabilized with a feedbacklinearizable input $u$

$$
u=\frac{m\left(-k_{d}^{z} \dot{z}-k_{p}^{z}\left(z-z_{r e f}\right)+g\right)}{\cos \theta \cos \phi}
$$

with $\theta$ and $\phi \neq \frac{\pi}{2}$. The rotorcraft flies so as to prevent these singularities. The global stabilization of the control strategy 
is not proven here since it is not the purpose of the paper (see [16]). From (25), we obtain

$$
\ddot{z}=-k_{d}^{z} \dot{z}-k_{p}^{z}\left(z-z_{\text {ref }}\right)
$$

Here $z$ is deduced from equation (7), where $w$ is calculated in the image plane, and $\dot{z}$ is deduced by numerical differentiation of equation (7). The gains $k_{p}^{z}$ and $k_{d}^{z}$ are chosen to ensure that the polynomial $s^{2}+k_{d}^{z} s+k_{p}^{z}$ is Hurwitz. Therefore, the altitude is stabilized to $z_{\text {ref }}$.

\section{B. Yaw subsystem}

Given equation (23), we can identify an independent system composed of two integrators in cascade. In order to ensure the stabilization of this sybsystem, we propose the following control strategy

$$
\tilde{\tau}_{\psi}=-k_{2}^{\psi} \dot{\psi}-k_{1}^{\psi} \psi
$$

The gains $k_{1}^{\psi}$ and $k_{2}^{\psi}$ are chosen appropriately so that the polynomial $s^{2}+k_{2}^{\psi} s+k_{1}^{\psi}$ is Hurwitz, and the yaw dynamics converges to zero.

\section{Longitudinal subsystem}

The longitudinal subsystem focuses on the $\dot{y}-\theta$ stabilization. Indeed, we will control the pitch and the longitudinal speed $\dot{y}$ to some references. Using (25) in (19) leads to

$$
m \ddot{y}=\left(v_{z}+g\right) \tan \theta
$$

where $v_{z}=-k_{d}^{z} \dot{z}-k_{p}^{z}\left(z-z_{\text {ref }}\right)$. Given that the rotorcraft flies close to the vertical, the angles are small and we can consider that $\tan \theta \approx \theta$. Also, since $z \rightarrow z_{\text {ref }}$ and $v_{z} \rightarrow 0$, the subsystem $\dot{y}-\theta$ can be rewritten as a linear subsystem

$$
\begin{aligned}
& \ddot{y}=g \theta \\
& \ddot{\theta}=\tilde{\tau}_{\theta}
\end{aligned}
$$

Considering $y_{1}=\frac{1}{g}\left(\dot{y}-V_{y}^{\text {ref }}\right)$, the longitudinal speed of the vehicle can be described with the following chain of integrators

$$
\begin{aligned}
& \dot{y}_{1}=y_{2} \\
& \dot{y}_{2}=y_{3} \\
& \dot{y}_{3}=\tilde{\tau}_{\theta}
\end{aligned}
$$

where $y_{1}$ is the error between the desired and the real speed, $y_{2}=\theta$ and $y_{3}=\dot{\theta}$. The hierarchical control is constructed by separating, in the previous subsystem, the rotational dynamics from the translational dynamics (low-level control and navigation control respectively). Considering $y_{2}$ as the control input for the translational dynamics, and applying a backstepping change of variables, we have

$$
\begin{aligned}
& \dot{y}_{1}=y_{2}^{\text {ref }}+\tilde{y}_{2} \\
& \dot{\tilde{y}}_{2}=\tilde{y}_{3} \\
& \dot{\tilde{y}}_{3}=\ddot{y}_{2}^{r e f}+\tilde{\tau}_{\theta}
\end{aligned}
$$

Here $y_{2}^{r e f}=\theta_{\text {ref }}, \tilde{y}_{2}=\theta-\theta_{\text {ref }}$, and $\tilde{y}_{3}=\dot{\theta}-\dot{\theta}_{\text {ref }}$. $\theta_{\text {ref }}$ is the reference angle that we will choose (compute) below by using the mean of the optical flow in the forward direction.
Because the low level autopilot (rotational control) works at highest rate than the navigation control, we can ignore the reference angle dynamics $\left(\dot{\theta}_{r e f}=\ddot{\theta}_{r e f}=0\right)$. Indeed, the dynamics of $\theta$ will converge faster than the navigation control. Then, we have

$$
\begin{aligned}
& \dot{y}_{1}=y_{2}^{r e f}+\tilde{y}_{2} \\
& \dot{\tilde{y}}_{2}=y_{3} \\
& \dot{y}_{3}=\tilde{\tau}_{\theta}
\end{aligned}
$$

We choose $y_{2}^{\text {ref }}=-k_{1}\left(\bar{V}_{O F_{y}}-\bar{V}_{O F_{y}}^{\text {ref }}\right)$ which leads to $y_{2}^{\text {ref }}=$ $-k_{p}^{y} y_{1}$, and $\tilde{\tau}_{\theta}=-k_{d}^{\theta} \dot{\theta}-k_{p}^{\theta}\left(\theta-\theta_{\text {ref }}\right)$. The closed loop system is represented by the polynomial: $s^{3}+k_{d}^{\theta} s^{2}+k_{p}^{\theta} s+k_{p}^{\theta} k_{p}^{y}$, which is Hurwitz if $k_{d}^{\theta}, k_{p}^{\theta}, k_{p}^{y}>0$ and $k_{d}^{\theta}>k_{p}^{y}$. Then, the subsystem $\dot{y}-\theta$ converges to the desired references.

\section{Lateral subsystem}

Assuming that the attitude is close to the origin and that the altitude has reached the desired value, the subsystem $x-$ $\phi$ can be expressed as

$$
\begin{array}{r}
\ddot{x}=-g \phi \\
\ddot{\phi}=\tilde{\tau}_{\phi}
\end{array}
$$

The lateral position of the vehicle can be described with the following chain of integrators

$$
\begin{aligned}
& \dot{x}_{1}=x_{2} \\
& \dot{x}_{2}=x_{3} \\
& \dot{x}_{3}=x_{4} \\
& \dot{x}_{4}=\tilde{\tau}_{\phi}
\end{aligned}
$$

We consider $\phi$ as the control input for the translational dynamics, then the chain of four integrators can be separated into two subsystems composed of two integrators for both of them. The implementation of a backstepping change of variables, gives

$$
\begin{aligned}
\dot{x}_{1} & =x_{2} \\
\dot{x}_{2} & =x_{3}^{r e f}+\tilde{x}_{3} \\
\dot{x}_{3} & =x_{4} \\
\dot{x}_{4} & =\tilde{\tau}_{\phi}
\end{aligned}
$$

where $x_{3}^{r e f}=\phi_{\text {ref }}$. This reference angle is computed by using $(\varsigma, \dot{\zeta})$ from equations (9) and (15) respectively. The reference angle dynamics are ignored, this assumption is achieved by using high gains in the low level autopilot. The image based variables $(\boldsymbol{\zeta}, \dot{\zeta})$ are used to stabilize the position, as follows

$$
\begin{aligned}
x_{3}^{r e f}=\phi_{\text {ref }} & =-k_{2}^{x} \dot{\zeta}-k_{1}^{x} \varsigma \\
\phi_{\text {ref }} & =-k_{d}^{x} \dot{x}-k_{p}^{x}\left(x-x_{b}\right)
\end{aligned}
$$

and

$$
\begin{aligned}
& \tilde{\tau}_{\phi}=-k_{d}^{\phi} \dot{x}_{4}-k_{p}^{\phi} \tilde{x}_{3} \\
& \tilde{\tau}_{\phi}=-k_{d}^{\phi} \dot{\phi}-k_{p}^{\phi}\left(\phi-\phi_{r e f}\right)
\end{aligned}
$$

The closed loop system is stable if $k_{p}^{\phi}, k_{d}^{\phi}, k_{p}^{x}, k_{d}^{x}>0, k_{d}^{\phi}>$ $k_{d}^{x}$, and $k_{p}^{\phi}>\frac{\left(k_{d}^{\phi}\right)^{2} k_{p}^{x}}{k_{d}^{x}\left(k_{d}^{\phi}-k_{d}^{x}\right)}$. Finally, the roll and the lateral displacement converge to their references. 


\section{EXPERIMENTAL APPLICATION}

\section{A. System Configuration}

Our experimental platform consists of an aerial vehicle (four-rotor rotorcraft), a base station and a HF video link (onboard transmitter, a ground receiver).

1) Aerial Vehicle: The rotorcraft was developed at Heudiasyc laboratory, in the University of Technology of Compiègne, in France. The distance (center to center) between two rotors is $40 \mathrm{~cm}$, and its total weight is approximately 750 grams. Figure 3 shows the prototype.

2) Base Station: A ground station was also developed. It consists of a desktop PC, a flight simulator joystick and a XBee ZB ZigBee PRO Radio Modem. The base station sends triggered information by the users to the aerial vehicle. The base station receives and saves all information needed to debug and understand the flight experiments and results.

3) Vision System: A high definition CTDM-5351 camera, with a resolution of $640 \times 480$ pixels is placed pointing downwards. The camera is connected to a $200 \mathrm{~mW}$ micro video and audio radio frequency transmitter. Images from the vision system are recovered by a 4-Antenna Diversity System Receiver. Receiver is connected to the ground station PC by means of a USB frame grabber. The frequency of the video transmission is at the rate of 30 frames per second.,

\section{B. Experimental Results}

The first experiment consists in hovering stabilization of the vehicle, in a specified zone of the road model. The experimental results can be seen in Figures 4, 5 and 6. We can remark from Figure 6 that the angles are always very small. A video showing this experiment can be found at http://www.youtube.com/watch?v= $\mathrm{xPb}-$ IHSsNIo\& feature=related
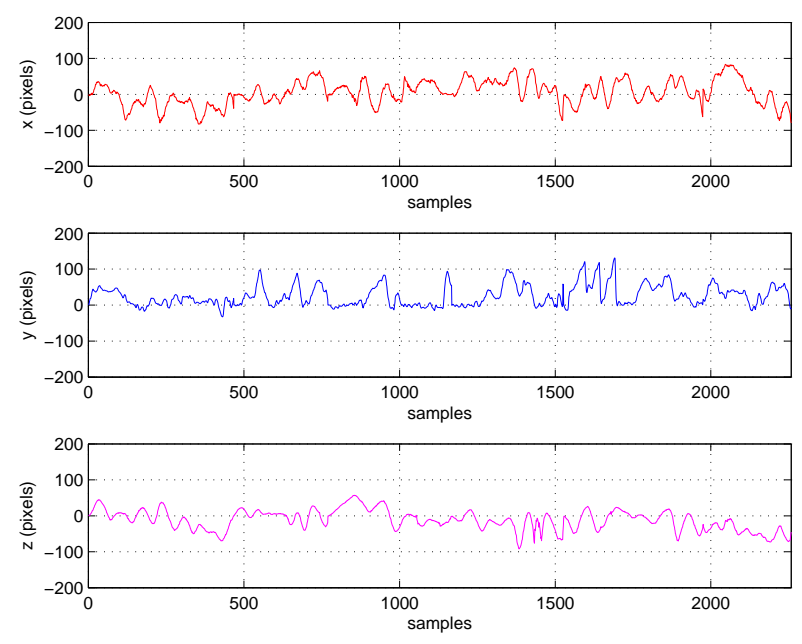

Fig. 4. Position results, hover stabilization

The set of Figures 7, 8, 9 shows the results (position, Euler angles and velocities respectively) for the speed regulation experiment. Figure 8 shows how the lateral speed is kept near zero while the forward speed converges to the desired
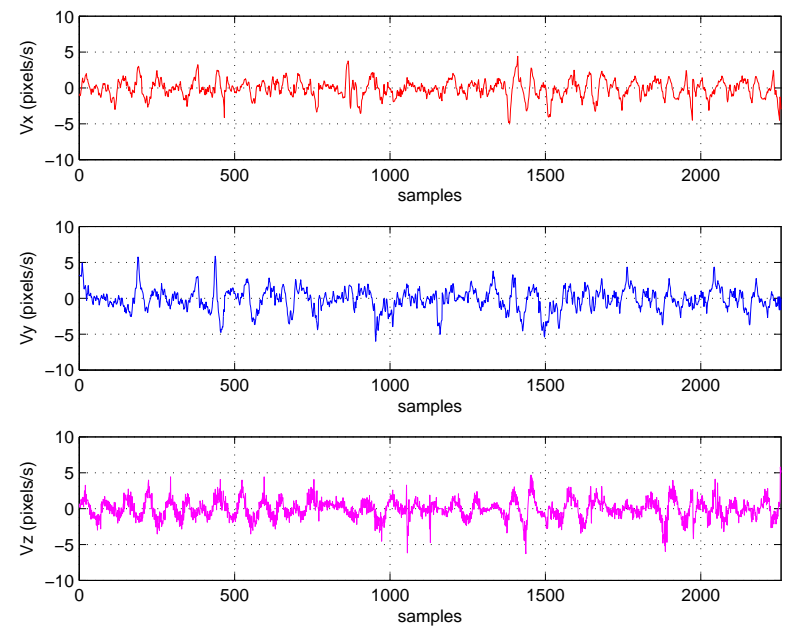

Fig. 5. Pseudo-velocities results, hover stabilization
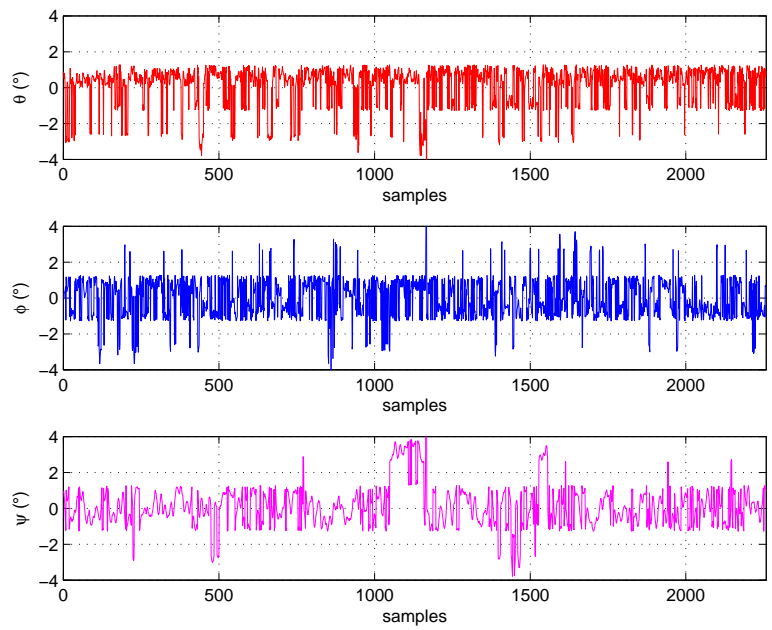

Fig. 6. Angles results, hover stabilization

value (which was set to 5), this value is not the real speed but the optical flow in the forward direction. It is important to remark that the angles converge also to desired values thanks to the control strategy. The altitude of the engine is also stabilized. In Figures 7 and 8, we can see that the altitude and the speed on $z$ only present small changes. A video of the UAV flying forward at constant speed can be found at http://www. youtube.com/watch? $\mathrm{v}=$ PpUW9a3S3GQ\& feature $=$ related

\section{CONClusions And Future Work}

A vision algorithm for altitude and position control, and speed regulation was proposed and tested in real time applications. The estimated information proves to be rich enough to enable navigation missions on a rotorcraft. Moreover, a road following type of mission was tested on a miniUAV quadrotor, using the proposed controller and vision algorithm. We have shown that the visual-based controller do not cause instability in the vehicle attitude. 

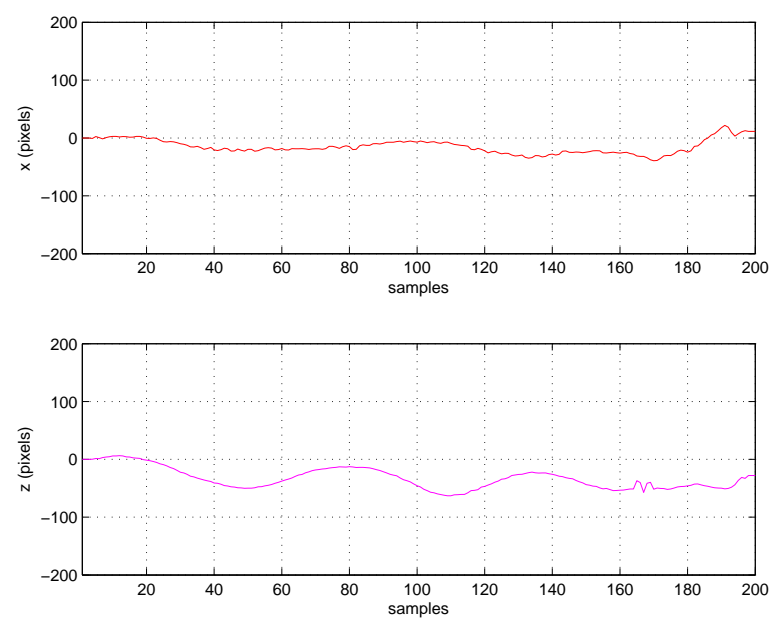

Fig. 7. Position results, speed regulation
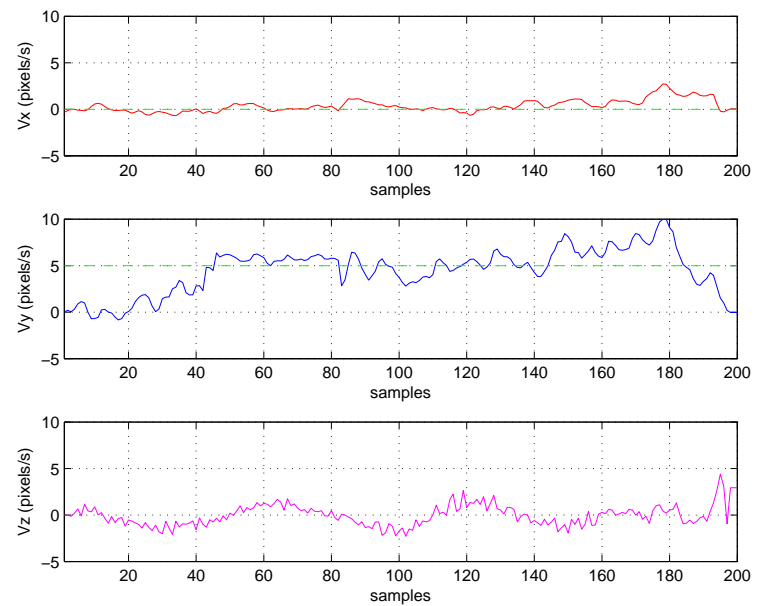

Fig. 8. Pseudo-velocities results, speed regulation
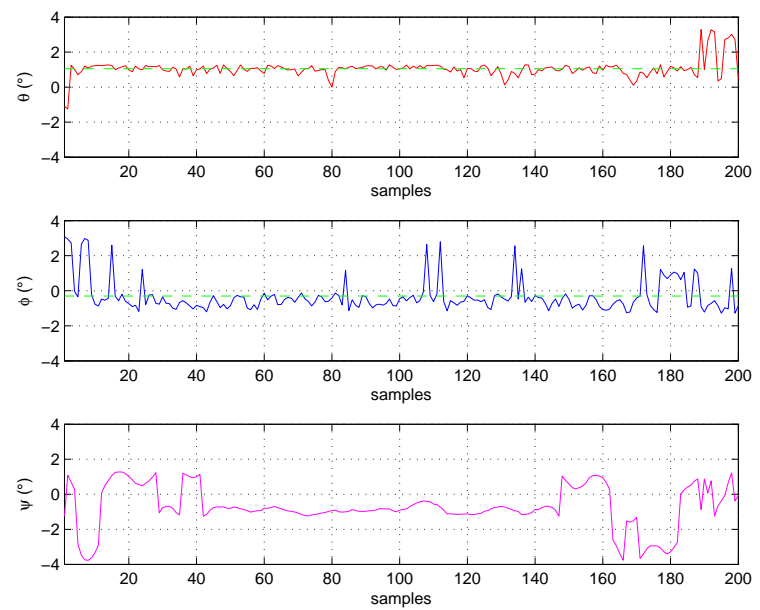

Fig. 9. Angles results, speed regulation
Future work will consist on detecting and avoiding frontal obstacles appearing on a frontal camera when constant speed forward flight mode is active. Also the speed regulation algorithm will be tested in combination with a new altitude estimator, in order to fly at constant speed in other environments.

\section{REFERENCES}

[1] F. Kendoul, D. Lara, I. Fantoni-Coichot, and R. Lozano, "Real-time nonlinear embedded control for an autonomous quadrotor helicopter," AIAA, Journal of Guidance, Control, and Dynamics, vol. 30, no. 4, pp. 1049-1061, July-August 2007.

[2] S. Salazar, J. Escareno, D. Lara, and R. Lozano, "Embedded control system for a four rotor UAV," International Journal of Adaptive Control and Signal Processing, vol. 21, no. 2-3, pp. 189-204, MarchApril 2007.

[3] A. Johnson, S. Montgomery, and L. Matthies, "Vision guided landing of an autonomous helicopter in hazardous terrain," in International Conference on Robotics and Automation, Barcelona,Spain, 2005, pp. 4470-4475.

[4] Z. Yu, K. Nonami, J. Shin, and D. Celestino, "3d vision based landign control of a small sacale autonomous helicopter," International Journal of Advanced Robotic Systems, vol. 4, no. 1, pp. 51-56, 2007.

[5] S. Hrabar, G. S.Sukhatme, P. Corke, K. Usher, and J. Roberts, "Combined optic-flow and stereo-based navigation of urban canyons for a UAV," in International Conference on Intelligent Robots and Systems, vol. 3, no. 4, Alberta,Canada, 2005, pp. 198-209.

[6] E. Rondon, S. Salazar, J. Escareno, and R. Lozano, "Vision-based position control of a two-rotor VTOL miniUAV," Journal of Intelligent and Robotic Systems, vol. 57, no. 1-4, pp. 49-64, January 2010.

[7] N. Guenard, T. Hamel, and R. Mahony, "A practical visual servo control for a unmanned aerial vehicle," IEEE Transactions on Robotics, vol. 24, no. 2, pp. 331-341, 2008.

[8] J. Chahl, M. Srinivasan, and S. Zhang, "Landing strategies in honeybees and applications to uninhabited airborne vehicles," Int. J. of Robotics Research, vol. 23, no. 2, pp. 101-110, February 2004.

[9] B. Herissé, F. Russotto, T. Hamel, and R. Mahony, "Hovering flight and vertical landing control of a VTOL unmanned aerial vehicle using optical flow," in IEEE/RSJ International Conference on Intelligent Robots and Systems, Nice, France, 2008.

[10] F. Ruffier and N. Franceschini, "Optic flow regulation: the key to aircraft automatic guidance," Robotics and Autonomous Systems, no. 50, pp. 177-194, 2005.

[11] M. Garrat and J. Chahl, "Vision-based terrain following for an unmanned rotorcraft," Journal of Field Robotics, vol. 25, no. 4-5, pp. 284-301, 2008.

[12] B. Herissé, T. Hamel, R. Mahony, and F. Russotto, "A nonlinear terrain-following controller for a VTOL unmanned aerial vehicle using translational optical flow," in IEEE Int. Conf. on Robotics and Automation, Kobe, Japan, May 2009.

[13] J. Serres, D. Dray, F. Ruffier, and N. Franceschini, "A vision-based autopilot for a miniature air vehicle: Joint speed control and lateral obstacle avoidance," Autonomous Robots, vol. 25, pp. 103-122, 2008.

[14] J.-C. Zufferey, A. Klaptocz, A. Beyeler, J.-D. Nicoud, and D. Floreano, "A 10 gram microflyer for vision-based indoor navigation," in IEEE International Conference on Intelligent Robots and Systems, Beijing,China, 2006.

[15] E. Rondon, I. Fantoni-Coichot, A. Sanchez, and G. Sanahuja, "Optical flow-based controller for reactive and relative navigation dedicated to a four rotor rotorcraft," in IEEE/RSJ International Conference on Intelligent Robots and Systems,IROS, St. Louis, USA, 10-15 Oct. 2009, pp. 684-689.

[16] P. Castillo, A. Dzul, and R. Lozano, "Real time stabilization and tracking of a four-rotor mini rotorcraft," IEEE Transactions on Control Systems Technology, vol. 12, no. 4, pp. 510-515, July 2004. 